Academic Platform Journal of Engineering and Science

\title{
Silindirik Sıvı Depolarının Dinamik Analizinin ACI 350 ve EUROCODE 8'e göre Karşılaştırılmalı olarak İrdelenmesi
}

\author{
*1 Badradine Ali, ${ }^{2}$ Adem Doğangün \\ ${ }^{1}$ Uludağ Üniversitesi., İnşaat Mühendisliği Bölümü, Görükle Kampüs, Bursa, Türkiye, \\ badradineali@yahoo.com, \\ ${ }^{2}$ Uludağ Üniversitesi., İnşaat Mühendisliği Bölümü, Görükle Kampüs, Bursa, Türkiye, \\ ademdogangun@gmail.com,
}

$\ddot{O} z$

Nüfusun sürekli artması ve sanayinin gelişmesi, başta su olmak üzere farklı sıvıların depolanma ihtiyacı, sıvı depolarının inşa edilmesini gerekli kılmaktadır. Diğer taraftan depoların dinamik davranışlarını daha gerçekçi olarak belirleyebilmek için çalışmalar da yapılmaktadır. Bu çalışmalardan uygun görülenler yönetmeliklere alınmaktadır. Bu çalışmanın amacı, sıvı depolarının depreme göre hesabını yapmak durumunda kalan ya da bu konuya ilgi duyan mühendislerin; sıvı depolarının dinamik yükler etkisindeki davranışını anlamalarına yardımcı olmak, bunlar için yönetmeliklerde öngörülen bağıntıları karşılaştırmalı olarak sunmak ve sıvı depolarının hesap ve tasarımını yapabilmelerine katkı sağlamaktır. Bu amaçla çalışmada, silindirik sıvı depolarının sismik analizi için ACI 350 ve EUROCODE 8 yönetmeliklerinde öngörülen hesap esasları ve parametrelerin belirlenmesinde kullanılan bağıntılar karşılaştırılmalı olarak irdelenmektedir.

\section{Comparative Investigation of Dynamic Analysis of Cylindrical Liquid Storage Tanks according to ACI 350 and EUROCODE 8}

\author{
*11 Badradine Ali, ${ }^{2}$ Adem Doğangün \\ ${ }^{1}$ Uludağ Üniversitesi., İnşaat Mühendisliği Bölümü, Görükle Kampüs, Bursa, Türkiye, badradineali@yahoo.com \\ ${ }^{2}$ Uludağ Üniversitesi., İnşaat Mühendisliği Bölümü, Görükle Kampüs, Bursa, Türkiye, ademdogangun@gmail.com
}

\begin{abstract}
The continuous increase in population and the development of industries necessitate the construction of liquid tanks for the storage of different liquids, especially water. Moreover, this study will be conducted to determine the dynamic behavior of liquid storage in more realistic manner. Likewise, the study have taken into consideration the usage of the appropriates international standard code. The aim of this study is to help engineers and those that are interested in this area to understand the behavior of liquid tanks under dynamic loads and to make comparism using the international standard code. The results obtained will contribute in terms the calculation and the design of liquid tanks. For this purpose, the relationship between the ACI 350 and EUROCODE 8 regulations for the seismic analysis of cylindrical liquid tanks and the formulas used in the determination of the parameters are compared.
\end{abstract}

Keywords: ACI 350, EUROCODE 8, impulsive masse, convective masse

*Sorumlu Yazar:Uludağ Üniversitesi., İnşaat Mühendisliği Bölümü, Görükle Kampüs, Bursa, Türkiye, badradineali@yahoo.com, 5442330292 


\section{GíRiş}

Bir yapıyı güvenli bir şekilde tasarlamak için, farklı yükleme koşulları altındaki davranışının ayrıntılı bir şekilde irdelenmesi gerekmektedir. Farklı yüklemelerden doğacak gerilmelerin ve şekil değiştirmelerin incelenmesi için farklı teorik, sayısal ve deneysel yöntemler bulunmaktadır. Deprem etkisi altında, sıvı depolarının projelendirilmesi, hem yükleme açısından hem de deponun özellikleri açısından önem arz etmektedir. Akaryakıt ve kimyasal sıvı depolarının tasarımında, özellikle deprem sonrasında çıkan yangınlar nedenleriyle daha hassas davranmak gerekmektedir. Çünkü önemli can kayıplarının yanında çevreye de telafisi zor olan zararlar verebilmektedir. Sıv1 depoları; ahşap, çelik veya betonarme olarak inşa edilebilmektedir. Betonarme ya da çelik olarak inşa edilen sıvı depolarının ekonomik ömürleri genellikle 40 ila 75 yıl arasında değişmektedir [13].

Bir sıvı deponun yapımına karar verildiğinde, hidrolik ve yapısal düşüncelerin bir arada değerlendirilmesi gerekmektedir. Çünkü bir depo; yapısal olarak mükemmel bir şekilde olsa da, hidrolik açısından yetersiz olabilir. Bunun tersinin olması da mümkündür. Özel mühendislik yapılarından olan betonarme depolar için verilen hesap esasları ve geçirimsizliğin sağlanması için alınan önlemler, yüzme havuzları için de geçerlidir.

\section{DEPOLARIN KULLANIM AMAÇLARI VE SINIFLANDIRILMASI}

Sıvı depoları genel olarak; dairesel, dikdörtgen veya kare kesitli olabilirler. Sıvı depoları için farklı açıdan yapılan sınıflandırmalar Şekil 1'deki şemada sunulmaktadır. Bu çalışmanın uygulamasına konu olan depo; sunulan depo sınıflarından zemine nazaran konumlarına göre yer üstü ve hazne geometrisine göre de silindirik olarak seçilmiştir.

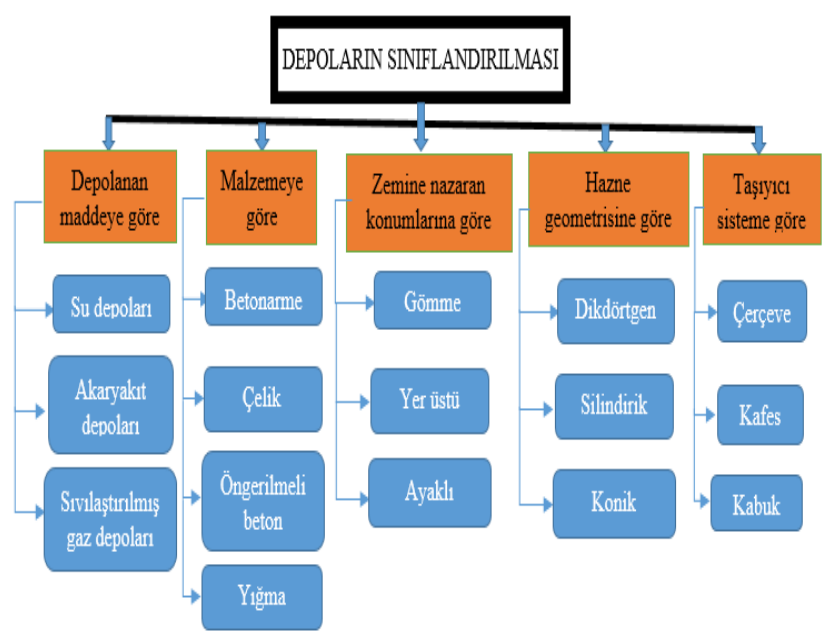

Şekil 1. Depoların sınıflandırılması [9]

\section{SIVI DEPOLARININ DINAMIK ANALIZİ İÇIN ÖNERILEN MEKANIK MODEL}

Sıvı depoları; dinamik bir etkiye maruz kaldığında, depo içindeki sıvının hareketi, asıl olarak iki kütlenin hareketi olarak modellenmektedir. Bunlardan ilki deponun yapisal kısmı ile birlikte hareket eden sıvı kütlesi (impuls kütlesi), ikincisi yapısal kısımdan bağımsız olarak hareket eden ve impuls kütlesine nazaran değişik periyotlarla salınım yapan sıvı kütlesi (salınım kütlelesi) dir. Bu model Şekil 2 de görülmektedir. Şekilde $R$ Silindirik depoların iç yarıçapını, $h$ sıvının yüksekliğini göstermektedir. Şekilde $m_{i}$ ve $m_{c}$ sırasıyla impuls ve salınım kütlelerini, $h_{i}$ ve $h^{\prime}{ }_{i}$ ise impuls kütlesinin ve devirici moment hesabında etkili impuls yüksekliklerini temsil etmektedir. Benzer şekilde, $h_{c}$ ve $h^{\text {' }}$ salınım kütlesinin ve devirici moment hesabında etkili salınım yüksekliğini ifade etmektedir.

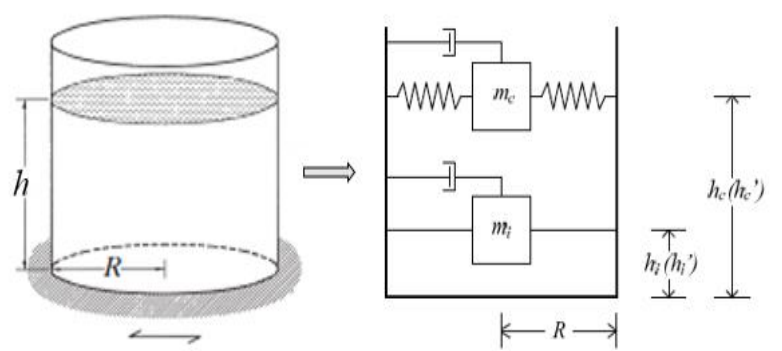

Şekil 2. Silindirik betonarme deponun dinamik modeli [6]

Deponun alt kısmındaki sıvının meydana getireceği etki impuls kuvveti, üst kısmındaki sıvının meydana getireceği etki ise salınım kuvveti ile temsil edilmektedir. Depo içindeki sıvı impuls kütlesinin depo duvarları ile birlikte hareket ettiği, salınım kütlesinin ise depo duvarlarından farklı olarak hareket ettiği belirtilebilir. İmpuls ve salınım titreşimi temsil eden toplanmış kütle kavramını kullanarak depoların dinamik analizi yapılabilmektedir. Bu modeller basit ve hızlı bir değerlendirme sağlamaktadır. Silindirik depolar için iki kütleli olarak tanımlanan bu model Housner tarafından yönetmelikleri girebilecek hale getirilmiştir.

\section{ACI 350 VE EUROCODE 8 DE DİKKATE ALINAN MODELLER}

Sıvı depolarının dinamik hesapları ile ilgili olarak ACI 350 ve EUROCODE 8 de toplanmış kütle yaklaşımı kullanılmaktadır. Bu bağlamda ilk olarak sıvı deposunun geometrik şekline göre sıvı deposu içindeki toplam kütle belirlenmekte, daha sonra bu kütleye bağlı olarak katsayılar kullanılarak impuls ve salınım kütleleri hesaplanmaktadır. Doğal olarak deponun boyutlarına bağlı olarak bu kütlelerin oranları da değişmektedir. En genel olarak çok sı̆̆ depolarda beklendiği gibi depo içindeki sıvının önemli bir kısmı salınım kütlesine katılmakla ve salınım kütlesi ön plana çıkabilmekte, derin depolarda ise impuls kütlesi daha etkin olabilmektedir. ACI 350 ve EUROCODE 8 de dikkate alınan 
kütlelerin değerleri Tablo 1 de bunların etkime yüksekliklerinin değerleri ise Tablo 2 de verilen bağıntılarla belirlenmektedir. Bu tabloda $R$ depo yarıçapını, $D$ depo çapını , $h$ sıvının yüksekliğini, $I_{1}$ değiştirilmiş bessel fonksiyonu, $I_{1}^{\prime}$ değiştirilmiş bessel fonksiyonun türevini, $\lambda_{n}$ birinci mertebeden bessel fonksiyonu, $v_{n} \bmod$ ve frekansa göre değişen bir katsayıyı göstermektedir.

EUROCODE 8 koduna göre, deponun bağıntılar deponun doluluk oranının fonksiyonuna göre belirlenmektedir, kütlelerin hesabından önce oranı hesaplayıp deponun sı̆ $\mathrm{m}$ derin mi olduğunu karar verilmektedir: $h / R<1.5$ ise depo siğ

$h / R>1.5$ ise depo derin.

Silindirik kesitli sı̆̆ ve derin depolardaki oranına göre impuls ve salınım kütlesinin değişimi Şekil 3 de, bu kütlelerin etkime yüksekliklerinin değişimi ise Şekil 4 de görülmektedir. Şekil 3 den görüldüğü gibi doluluk oranının çok küçük değerleri için salınım kütlesi oranı çok büyük olurken, bu oran 0.8 civarında her iki kütle değeri birbirine eşit olmakta, bu değerden sonra da artık impuls kütlesinin oranı artarak devam etmektedir.

Tablo 1. Silindirik depo içindeki sıvı kütlelerinin ACI 350 ve EUROCODE 8'e göre belirlenmesi

\begin{tabular}{|l|c|c|}
\hline Yönetmelikler & ACI 350.3-06 & EUROCODE 8 \\
\hline Toplam sıvı kütlesi & $m_{l}=\pi \cdot R^{2} \cdot h \cdot \rho_{l}$ & $m_{l}=\pi \cdot R^{2} \cdot h \cdot \rho_{l}$ \\
\hline İmpuls kütlesi oranı & $\frac{m_{i}}{m_{l}}=\frac{\tanh \left(0.866\left(\frac{D}{h}\right)\right)}{0.866\left(\frac{D}{h}\right)}$ & $\frac{m_{i}}{m_{l}}=2\left(\frac{h}{R}\right) \sum_{n=0}^{\infty} \frac{I_{1}\left[v_{n} /(h / R)\right]}{v_{n}^{3} I_{1}\left[v_{n} /(h / R)\right]}$ \\
\hline Salınım kütlesi oranı & $\frac{m_{c}}{m_{l}}=0.230 \times\left(\frac{D}{h}\right) \times \tanh \left(3.68 \frac{h}{D}\right)$ & $\frac{m_{c}}{m_{l}}=\frac{2 \tanh \left[\lambda_{n}(h / R)\right]}{\lambda_{n}(h / R)\left(\lambda_{n}{ }^{2}-1\right)}$ \\
\hline
\end{tabular}

Tablo 2. ACI 350 ve EUROCODE 8'e göre sıvıya ilişkin etkili yüksekliklerin belirlenmesi

\begin{tabular}{|c|c|c|}
\hline Yönetmelikler & ACI 350.3-06 & EUROCODE 8 \\
\hline $\begin{array}{|lrl|}\text { İmpuls } & \text { kütlesi } & \text { etkime } \\
\text { yüksekliği oranı } & \end{array}$ & $\begin{array}{c}\frac{D}{h} \geq 1.333 \Rightarrow \frac{h_{i}}{h}=0.375 \\
\text { Ĕger } \frac{D}{h}<1.333 \Rightarrow \\
\frac{h_{i}}{h}=0.5-0.09375\left(\frac{h}{D}\right)\end{array}$ & $\frac{h_{i}}{h}=\frac{\sum_{n=0}^{\infty} \frac{(-1)^{n} I_{1}\left[v_{n} /(h / R)\right]}{v_{n}{ }^{4} I_{1}^{\prime}\left[v_{n} /(h / R)\right]}\left[v_{n}(-1)^{n}-1\right]}{\sum_{n=0}^{\infty} \frac{I_{1}\left[v_{n} /(h / R)\right]}{v_{n}^{3} I_{1}^{\prime}\left[v_{n} /(h / R)\right]}}$ \\
\hline 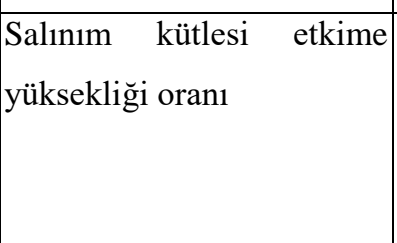 & $\frac{h_{c}}{h}=1-\frac{\cosh \left[3.68 \times \frac{h}{D}\right]-1}{3.68 \times \frac{h}{D} \times \sinh \left(3.68 \frac{h}{D}\right)}$ & $\frac{h_{c}}{h}=1+\frac{1-\cosh \left[\lambda_{n}(h / R)\right]}{\lambda_{n}(h / R) \sin \left[\lambda_{n}(h / R)\right]}$ \\
\hline \begin{tabular}{|l} 
Devirici moment hesabında \\
impuls kütlesi etkime \\
yüksekliği oranı
\end{tabular} & $\begin{array}{c}\frac{D}{h}<0.75 \Rightarrow \frac{h_{i}^{\prime}}{h}=0.45 \\
\text { Ĕger } \frac{D}{h} \geq 0.75 \Rightarrow\end{array}$ & $\frac{h_{i}^{\prime}}{h}=\frac{\frac{1}{2}+2\left(\frac{h}{R}\right) \sum_{n=0}^{\infty} \frac{v_{n}+2(-1)^{n+1} I_{1}\left[v_{n} /(h / R)\right]}{2\left(\frac{h}{R}\right) \sum_{n=0}^{\infty} \frac{v_{n}{ }^{4} I_{1}^{\prime}\left[v_{n} /(h / R)\right]}{I_{n}\left[v_{n} /(h / R)\right]}}}{I_{1}^{\prime}\left[v_{n} /(h / R)\right]}$ \\
\hline
\end{tabular}




\begin{tabular}{|l|c|c|}
\hline & $\frac{h_{i}^{\prime}}{h}=\frac{0.866 \times \frac{D}{h}}{2 \tanh \left(0.866 \times \frac{D}{h}\right)}-\frac{1}{8}$ & \\
\hline $\begin{array}{l}\text { Devirici moment hesabında } \\
\text { salınım kütlesi etkime } \\
\text { yüksekliği oranı }\end{array}$ & $\frac{h_{c}^{\prime}}{h}=1-\frac{\cosh \left[3.68 \times \frac{h}{D}\right]-2.01}{3.68\left(\frac{h}{D}\right) \sinh \left(3.68 \frac{h}{D}\right)}$ & $\frac{h_{c}^{\prime}}{h}=1+\frac{2-\cosh \left[\lambda_{n}(h / R)\right]}{\lambda_{n}(h / R) \sin \left[\lambda_{n}(h / R)\right]}$ \\
\hline
\end{tabular}

Şekil 4 den görüldüğü gibi impuls ve salınım kütlelerin etkime yüksekliklerinin değişimleri de doluluk oranının küçük değerleri için birbirinden çok farklı olmakta, ancak bu oranın 1 değerinden itibaren aralarındaki farklar azalmakta ve yataya yakın bir şekilde ilerlemektedir.

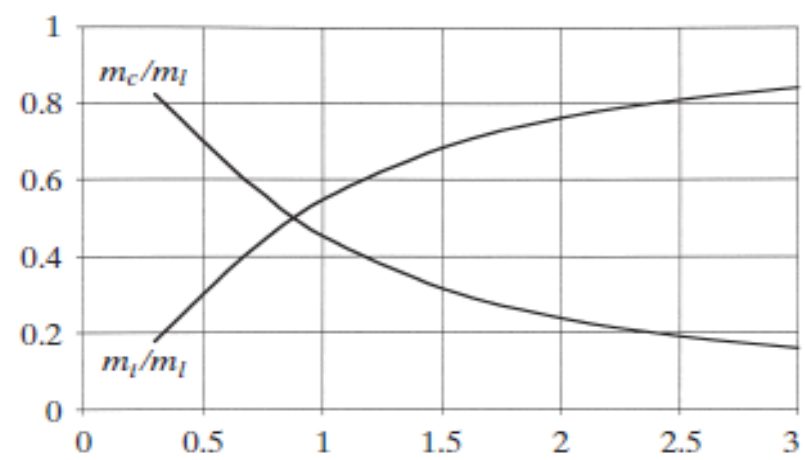

Şekil 3. $h / R$ oranına göre $m_{i} / m_{l}$ ve $m_{c} / m_{l}$ oranları [4]

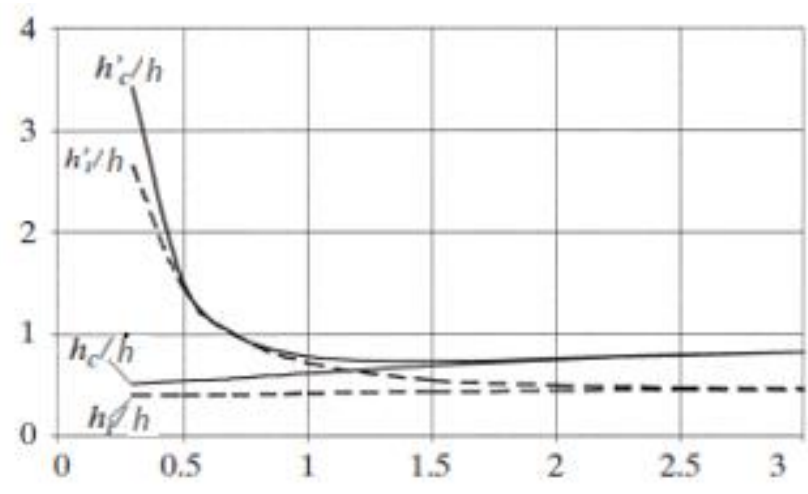

Şekil 4. $h / R$ oranına göre $h_{i} / h, h_{c} / h, h^{\prime} i / h, h^{\prime} d h$ oranları [4]

EUROCODE 8'e göre $h / R$ oranına göre, $C_{i}$ ve $C_{c}$ katsayıların, kütlelerin ve etkime yüksekliklerinin değerleri Tablo 3 de sunulmaktadır. $C_{i}$ ve $C_{c}$ katsayılarının $h / R$ oranına göre değişimleri grafik olarak Şekil 5 de sunulmaktadır. $\mathrm{Bu}$ şekilden görüldüğ̈̈ gibi $C_{i}$ katsayısındaki değişim daha fazla olmaktadır.

Tablo 3. Eurocode 8 'e göre $h / R$ oranına göre dinamik parametrelerin değerleri

\begin{tabular}{|c|c|c|c|c|c|c|c|c|}
\hline$h / R$ & $C_{i}$ & $C_{c}\left(\mathrm{~s} / \mathrm{m}_{1} / 2\right)$ & $m_{i} / m_{l}$ & $m_{c} / m_{l}$ & $h_{i} / h$ & $h_{c} / h$ & $h_{i}{ }_{i} / h$ & $h_{c}{ }_{c} / h$ \\
\hline 0,3 & 9,28 & 2,09 & 0,176 & 0,824 & 0,400 & 0,521 & 2,640 & 3,414 \\
\hline 0,5 & 7,74 & 1,74 & 0,300 & 0,700 & 0,400 & 0,543 & 1,460 & 1,517 \\
\hline 0,7 & 6,97 & 1,60 & 0,414 & 0,586 & 0,401 & 0,571 & 1,009 & 1,011 \\
\hline 1,0 & 6,36 & 1,52 & 0,548 & 0,452 & 0,419 & 0,616 & 0,721 & 0,785 \\
\hline 1,5 & 6,06 & 1,48 & 0,686 & 0,314 & 0,439 & 0,690 & 0,555 & 0,734 \\
\hline 2,0 & 6,21 & 1,48 & 0,763 & 0,237 & 0,448 & 0,751 & 0,500 & 0,764 \\
\hline 2,5 & 6,56 & 1,48 & 0,810 & 0,190 & 0,452 & 0,794 & 0,480 & 0,796 \\
\hline 3,0 & 7,03 & 1,48 & 0,842 & 0,158 & 0,453 & 0,825 & 0,472 & 0,825 \\
\hline
\end{tabular}




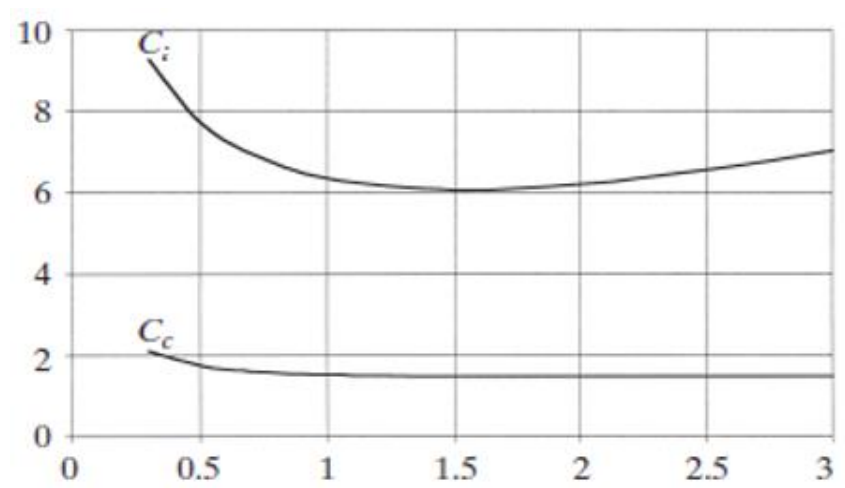

Silindirik bir deponun titreşimi için impuls ve salınım periyotları olmak üzere iki farklı periyot belirlenmektedir. $\mathrm{Bu}$ periyotların belirlenmesi için gerekli olan bağıntılar Tablo 4 de sunulmaktadır. Bu tablodaki semboller: $E$ elastisite modülü, $C_{w}$ ve $C_{l}$ periyodun hesabında kullanılan katsayılar, $g$ yerçekimi ivmesi, $\gamma_{c}$ beton yoğunluğu, $C_{c}$ periyodun hesabında kullanılan katsayılar, $\rho$ siv1 yoğunluğu, $S$ duvar kalınlığı, $C_{i}$ periyodun hesabında kullanılan katsayı.

Şekil 5. $h / R$ oranına göre $C_{i}$, $C_{c}$ katsayılarının değişimi [4]

Tablo 4. ACI 350 ve EUROCODE 8'e göre sıvıya ilişkin periyotların belirlenmesi

\begin{tabular}{|l|c|c|}
\hline Yönetmelikler & ACI 350.3-06 & EUROCODE 8 \\
\hline İmpuls periyodu & $C_{l}=C_{w} \times \sqrt{\frac{t_{w}}{10 \times R}}$ ve & $T_{i}=C_{i} \times \frac{\sqrt{\rho} \times h}{\sqrt{S / R} \times \sqrt{E}}$ \\
& $w_{i}=C_{l} \times \frac{1}{h} \sqrt{10^{3} E g / \gamma_{c}}$ olmak üzere & \\
\hline Salınım periyodu & $T_{i}=\frac{2 \pi}{w_{i}}$ & \\
hesab1 $\left(T_{c}\right)$ & $\lambda=\sqrt{3.68 g \tanh \left[3.68\left(\frac{h}{D}\right)\right]}$ olmak üzere & $T_{c}=C_{c} \times \sqrt{R}$ \\
& $T_{c}=\frac{2 \pi}{\lambda} \times \sqrt{D}$ & \\
\hline
\end{tabular}

Bir deponun toplam sismik tepkisi için doğal titreşim periyotları, kesme kuvveti ve devirici moment açısından analiz edilmesi gerekmektedir. ACI 350 ve EUROCODE 8'e göre kesme kuvveti hesabı Tablo 5 de, moment hesabı ise Tablo 6 da sunulmaktadir.

Tablo 5. ACI 350 ve EUROCODE 8'e göre depremde oluşabilecek kesme kuvveti hesabı (V)

\begin{tabular}{|c|c|}
\hline ACI 350.3-06 & EUROCODE 8 \\
\hline$V=\sqrt{\left(P_{i}+P_{w}+P_{r}\right)^{2}+P_{c}^{2}}$ & $V=\left(m_{i}+m_{w}+m_{r}\right) \times S_{e}\left(T_{i}\right)+m_{c} S_{e}\left(T_{c}\right)$ \\
İmpuls kuvvetinin hesabı $\left(P_{i}\right)$ & $\begin{array}{c}\text { Duvar kütlesi hesab1 } \\
=2 \times \pi \times R \times H \times t_{w} \times \rho_{c} \\
P_{i}=Z . S . I . C_{i} \times \frac{m_{i}}{R_{W_{i}}}\end{array}$ \\
$P_{w}=Z . S . I . \varepsilon . C_{i} \times \frac{m_{w}}{R_{W_{i}}}$ & $\begin{array}{c}\text { Kapak kütlesi hesabi } \\
m_{r}=\pi \times R^{2} \times t_{r} \times \rho_{c} \\
\end{array}$ \\
\hline
\end{tabular}


Çatı kütlesi etkisiyle meydana gelen kuvvet hesabı $\left(P_{r}\right)$

$$
P_{r}=\text { Z.S.I.C }{ }_{i} \times \frac{m_{r}}{R_{W_{i}}}
$$

Salınım kuvvetinin hesabı $\left(P_{c}\right)$

$$
P_{c}=Z . S . I . C_{c} \times \frac{m_{c}}{R_{W_{c}}}
$$

ACI 350 de verilen tablolardan alınacak katsayılar (Z ,S,I,

$$
\left.R_{W_{i}}, R_{W_{c}}\right)
$$

Duvar yükü etkisiyle oluşacak kuvvet hesabında kullanılan

$$
\begin{gathered}
\varepsilon \leq 1.0 \text { katsayı } \\
\varepsilon=\left[0.0151\left(\frac{D}{h}\right)^{2}-0.1908\left(\frac{D}{h}\right)+1.021\right] \\
C_{i} \text { impuls periyoduna göre hesaplanır,eğer }
\end{gathered}
$$

$$
\begin{gathered}
T_{i} \leq 0.31 s \text { ise } C_{i}=2.5 \\
T_{i} \geq 0.31 s \text { ise } C_{i}=\frac{1.25}{T_{i}^{2 / 3}}
\end{gathered}
$$

\begin{tabular}{|c|c|c|}
\hline Yönetmelikler & ACI 350.3-06 & EUROCODE 8 \\
\hline $\begin{array}{l}\text { Eğilme } \\
\text { momenti }\left(M_{b}\right)\end{array}$ & $\begin{array}{c}M_{b}=\sqrt{\left(M_{i}+M_{w}+M_{r}\right)^{2}+M_{c}^{2}} \\
\text { İmpuls kuvetinden oluşan impuls momenti } \\
\qquad M_{i}=P_{i} \times h_{i} \\
\text { Duvar kütlesinden meydana gelen duvar } \\
\text { momenti } \\
M_{W}=P_{W} \times h_{W} \\
\text { Çatı kütlesinden meydana gelen çatı } \\
\text { momenti } \\
M_{r}=P_{r} \times h_{r}\end{array}$ & $M_{b}=\left(m_{i} h_{i}+m_{w} h_{w}+m_{r} h_{r}\right) \cdot S_{e}\left(T_{i}\right)+m_{c} h_{c} \cdot S_{e}\left(T_{c}\right)$ \\
\hline
\end{tabular}

$C_{c}$ salınım periyoduna göre hesaplanır.

$$
T_{c} \geq 2.4 s ; C_{c}=\frac{6}{T_{c}^{2}}
$$

$$
\begin{gathered}
0 \leq T_{n} \leq T_{B}: \\
S_{e}\left(T_{n}\right)=a_{g} \cdot S \cdot\left[1+\frac{T_{n}}{T_{B}} \cdot(\eta \cdot 2,5-1)\right] \\
T_{B} \leq T_{n} \leq T_{C}: \\
S_{e}\left(T_{n}\right)=a_{g} \cdot S \cdot \eta \cdot 2,5 \\
T_{C} \leq T_{n} \leq T_{D}: \\
S_{e}\left(T_{n}\right)=a_{g} \cdot S \cdot \eta \cdot 2,5 \cdot\left[\frac{T_{C} \cdot T_{D}}{T_{n}^{2}}\right] \\
T_{D} \leq T_{n} \leq 0.4 s \\
\left.T_{n}\right)=a_{g} \cdot S \cdot \eta \cdot 2,5 \cdot\left[\frac{T_{C}}{T_{n}}\right]
\end{gathered}
$$

İvme hesabında alınan değerler EUROCODE 8 Part I den kullanılmıştır.

Tablo 6. ACI 350 ve EUROCODE 8'e göre sıvıya ilişkin moment ve yüksekliklerin belirlenmesi 


\begin{tabular}{|c|c|c|}
\hline & $\begin{array}{l}\text { Salınım kuvvetinden oluşan salınım } \\
\text { momenti } \\
M_{c}=P_{c} \times h_{c}\end{array}$ & \\
\hline \begin{tabular}{|l} 
Deprem \\
esnasında \\
oluşabilecek \\
devirici momenti \\
$\left(M_{o}\right)$
\end{tabular} & $\begin{array}{c}M_{o}=\sqrt{\left(M_{i}^{\prime}+M_{W}+M_{r}\right)^{2}+M_{c}^{\prime 2}} \\
\text { Devirici momenti hesabında kullanılan } \\
\text { impuls momenti } \\
\qquad M_{i}^{\prime}=P_{i} \times h_{i}^{\prime} \\
\text { Devirici momenti hesabında kullanılan } \\
\text { salınım momenti } \\
\qquad M_{c}^{\prime}=P_{c} \times h_{c}^{\prime} \\
\text { Duvar kütlesinden meydana gelen duvar } \\
\text { momenti } \\
M_{W}=P_{W} \times h_{W} \\
\text { Çatı kütlesinden meydana gelen çatı } \\
\text { momenti } \\
M_{r}=P_{r} \times h_{r}\end{array}$ & $M_{o}=\left(m_{i} h_{i}^{\prime}+m_{w} h_{w}+m_{r} h_{r}\right) \cdot S_{e}\left(T_{i}\right)+m_{c} h_{c}^{\prime} \cdot S_{e}\left(T_{c}\right)$ \\
\hline \begin{tabular}{|l|} 
Maksimum \\
oluşabilecek \\
dalga yüksekliği \\
$\left(d_{\max }\right)$
\end{tabular} & $d_{\max }=\left(\frac{D}{2}\right) I \times \eta_{c} \times \frac{1.226 S_{D}}{T_{c}^{2}}$ & $d_{\max }=R \times \frac{S_{e}\left(T_{c}\right)}{g}$ \\
\hline
\end{tabular}

\section{SAYISAL UYGULAMA}

Bu bölümde pratik uygulama için EUROCODE 8'e göre rijit ve esnek, ACI 350 ye göre esnek çözüm yapılmıştır. Dördüncü (4) deprem bölgeye göre analiz edilmiştir ( her iki standart için yer ivmesi ve yapı davranış katsayısı aynı seçilmiştir).

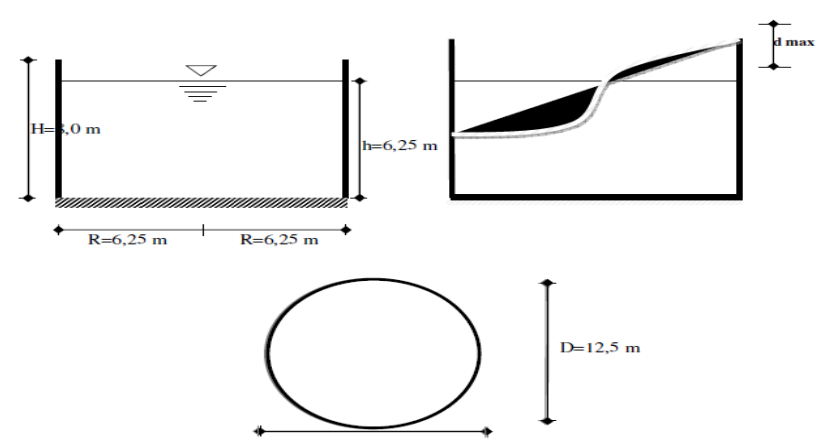

Şekil 6. Kullanılan depo modeli
Table 7. Verilen parametre

\begin{tabular}{|c|c|}
\hline ACI 350.3-06 & EUROCODE 8 \\
\hline$E=21.10^{3} \mathrm{~N} / \mathrm{m}^{2}$ & $h=6.25 m$ \\
\hline$v=0.17$ & $R=6.25 m$ \\
\hline$t_{w}=0.5 m$ & $H=8 m$ \\
\hline$g=9.81 \mathrm{~m} / \mathrm{s}^{2}$ & $\gamma_{l}=1000 \mathrm{k}_{g} / \mathrm{m}^{3}$ \\
\hline$Z=1.226$ & $a_{g R}=0.1 \mathrm{~g}$ \\
\hline
\end{tabular}


Tablo 8. ACI 350 ve EUROCODE 8'e göre sıvıya ilişkin kütle,etkili yükseklik ve salınım periyodun hesabı

\begin{tabular}{|c|c|c|}
\hline Yönetmelikler & ACI 350.3-06 & EUROCODE 8 \\
\hline Toplam sıvı kütlesi & $\begin{array}{c}m_{l}=\pi \times \frac{12.5^{2}}{4} \times 6.25 \times 1000 \\
m_{l}=766601.563 k_{g}\end{array}$ & $\begin{array}{c}m_{l}=\pi \times 6.25^{2} \times 6.25 \times 1000 \\
m_{l}=766601.563 k_{g}\end{array}$ \\
\hline İmpuls kütlesi oranı & $\begin{array}{c}\frac{m_{i}}{m_{l}}=\frac{\tanh \left[0.866\left(\frac{12.5}{6.25}\right)\right]}{0.866\left(\frac{12.5}{6.25}\right)} \\
m_{i}=415611.356 k_{g}\end{array}$ & $\begin{array}{c}h / R=\frac{6.25}{6.25} \Rightarrow h / R=1 \\
\frac{m_{i}}{m_{l}}=0.548 \Rightarrow m_{i}=0.548 m_{l} \\
m_{i}=0.548 \times 766601.563 \\
m_{i}=420097.656 k_{g}\end{array}$ \\
\hline Salınım kütlesi oranı & $\begin{array}{c}\frac{m_{c}}{m_{l}}=0.230\left(\frac{12.5}{6.25}\right) \cdot \tanh \left[3.68\left(\frac{6.25}{12.5}\right)\right] \\
m_{c}=352636.434 k_{g}\end{array}$ & $\begin{array}{c}\frac{m_{c}}{m_{l}}=0.452 \Rightarrow m_{c}=0.452 m_{l} \\
m_{c}=0.452 \times 766601.563 \\
m_{c}=346503.907 k_{g}\end{array}$ \\
\hline $\begin{array}{l}\text { impuls kütlesi etkili } \\
\text { yükseklik hesabı }\end{array}$ & $\begin{array}{c}\text { Depoda } \frac{D}{h}>1.333 \Rightarrow \frac{h i}{h}=0.375 \\
h_{i}=0.375 \times 6.25 \\
h_{i}=2.344 \mathrm{~m}\end{array}$ & $\begin{array}{c}\frac{h_{i}}{h}=0.419 \Rightarrow h_{i}=0.419 h \\
h_{i}=0.419 \times 6.25 \\
h_{i}=2.619 \mathrm{~m}\end{array}$ \\
\hline $\begin{array}{l}\text { Salınım kütlesi etkili } \\
\text { yükseklik hesabı }\end{array}$ & $\begin{array}{c}\frac{h_{c}}{h}=1-\frac{\cosh \left[3.68\left(\frac{6.25}{12.5}\right)\right]-1}{3.68\left(\frac{6.25}{12.5}\right) \cdot \sinh \left[3.68\left(\frac{6.25}{12.5}\right)\right]} \\
\frac{h_{c}}{h}=0.602 \Rightarrow h_{c}=0.602 h \Rightarrow h_{c}=3.763 m\end{array}$ & $\begin{array}{c}\frac{h_{c}}{h}=0.616 \Rightarrow h_{c}=0.616 h \\
h_{c}=0.616 \times 6.25 \\
h_{c}=3.850 m\end{array}$ \\
\hline 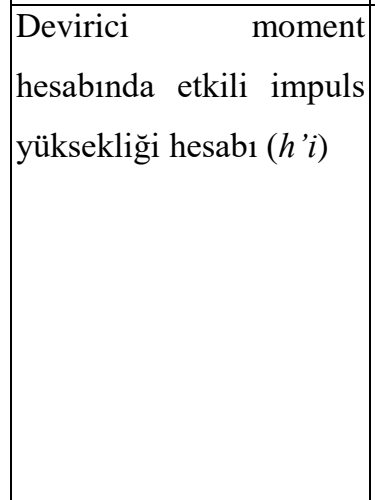 & $\begin{array}{c}\text { Depoda } \frac{D}{h} \geq 0.75 \\
\frac{h_{i}^{\prime}}{h}=\frac{0.866\left(\frac{12.5}{6.25}\right)}{2 \tanh \left[\left(0.866\left(\frac{12.5}{6.25}\right)\right]\right.}-\frac{1}{8} \\
\frac{h_{i}^{\prime}}{h}=0.797 \Rightarrow h_{i}^{\prime}=4.981 \mathrm{~m}\end{array}$ & $\begin{array}{c}\frac{h_{i}^{\prime}}{h}=0.721 \Rightarrow h_{i}^{\prime}=0.721 \times h \\
h_{i}^{\prime}=0.721 \times 6.25 \\
h_{i}^{\prime}=4.506 m\end{array}$ \\
\hline
\end{tabular}




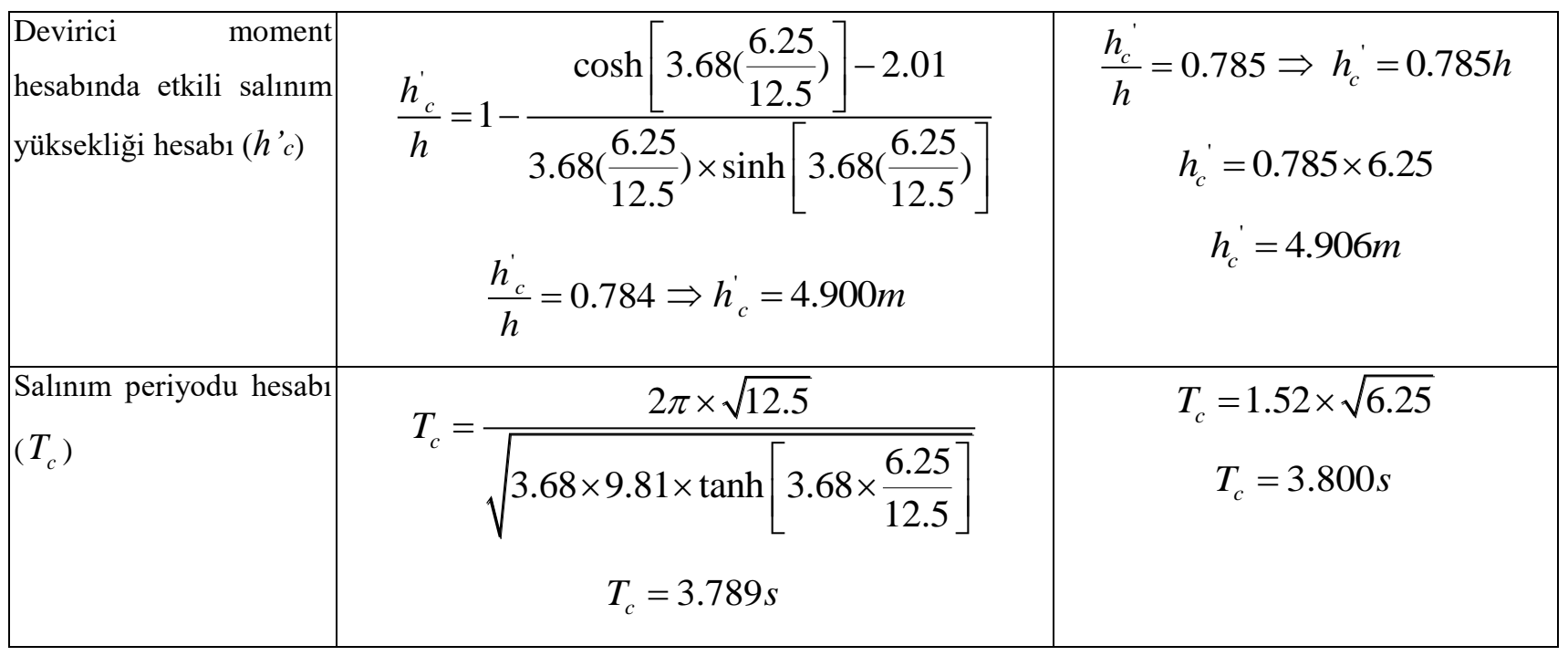

Tablo 9. ACI 350 ve EUROCODE 8'e göre sıvıya ilişkin impuls periyodun hesabı $\left(T_{i}\right)$

\begin{tabular}{|c|c|}
\hline ACI 350.3-06 & EUROCODE 8 \\
\hline $\begin{array}{c}T_{i}=\frac{2 \pi}{\omega_{i}} \\
C_{l} \text { Katsayısı hesabında kullanıllan } C_{w} \text { katsayının hesabı : } \\
C_{w}=9.375 .10^{-2}+0.2039\left(\frac{6.25}{12.5}\right)-0.1034\left(\frac{6.25}{12.5}\right)^{2} \\
-0.1253\left(\frac{6.25}{12.5}\right)^{3}+0.1267\left(\frac{6.25}{12.5}\right)^{4}-3.186 .10^{-2}\left(\frac{6.25}{12.5}\right)^{5} \\
C_{w}=0.1611099 \\
C_{l} \text { Katsayının hesabi: } \\
C_{l}=0.1611099 \sqrt{\frac{0.5}{10 \times 6.25}} \\
C_{l}=0.0144 \\
\text { Açısal hız hesabı } \\
T_{i}=0.0144 \times \frac{1}{6.25} \sqrt{\frac{10^{3} \times 21.10^{3} \times 9.81}{2.4}} \\
\omega_{i}=21.346 \\
T_{i}=0.300 \mathrm{~s}\end{array}$ & \begin{tabular}{|c}
$\begin{array}{c}\text { Depo rijit olarak kabul edildiğinde sıv1 deposu deprem } \\
\text { esnasında zemin hareketini taklit edecektir. } \\
\text { Dördüncü deprem bölgesi kabulune göre zemin ivmenin } \\
\text { hesabı : }\end{array}$ \\
$\gamma_{l} \geq 1 \Rightarrow a_{g R}=0.1 \mathrm{~g}$ \\
$a_{g R}=0.1 \times 9.81$ \\
$a_{g R}=0.981$ \\
$a_{g}=\gamma_{l} \times a_{g R}$ \\
$a_{g}=1.25 \times 0.981$ \\
$a_{g}=1.226 m / s^{2}$ \\
Depo esnek olarak kabul edildiği zaman impuls \\
periyodun hesabını yapılmalı: \\
$T_{i}=6.36 \times \frac{\sqrt{1} \times 6.25}{\sqrt{\frac{0.5}{6.25}} \times \sqrt{21.10^{3}}}$ \\
$T_{i}=0.306 \mathrm{~s}$
\end{tabular} \\
\hline
\end{tabular}


Tablo 10. Deprem esnasında oluşabilecek kesme kuvveti hesabı $(V)$

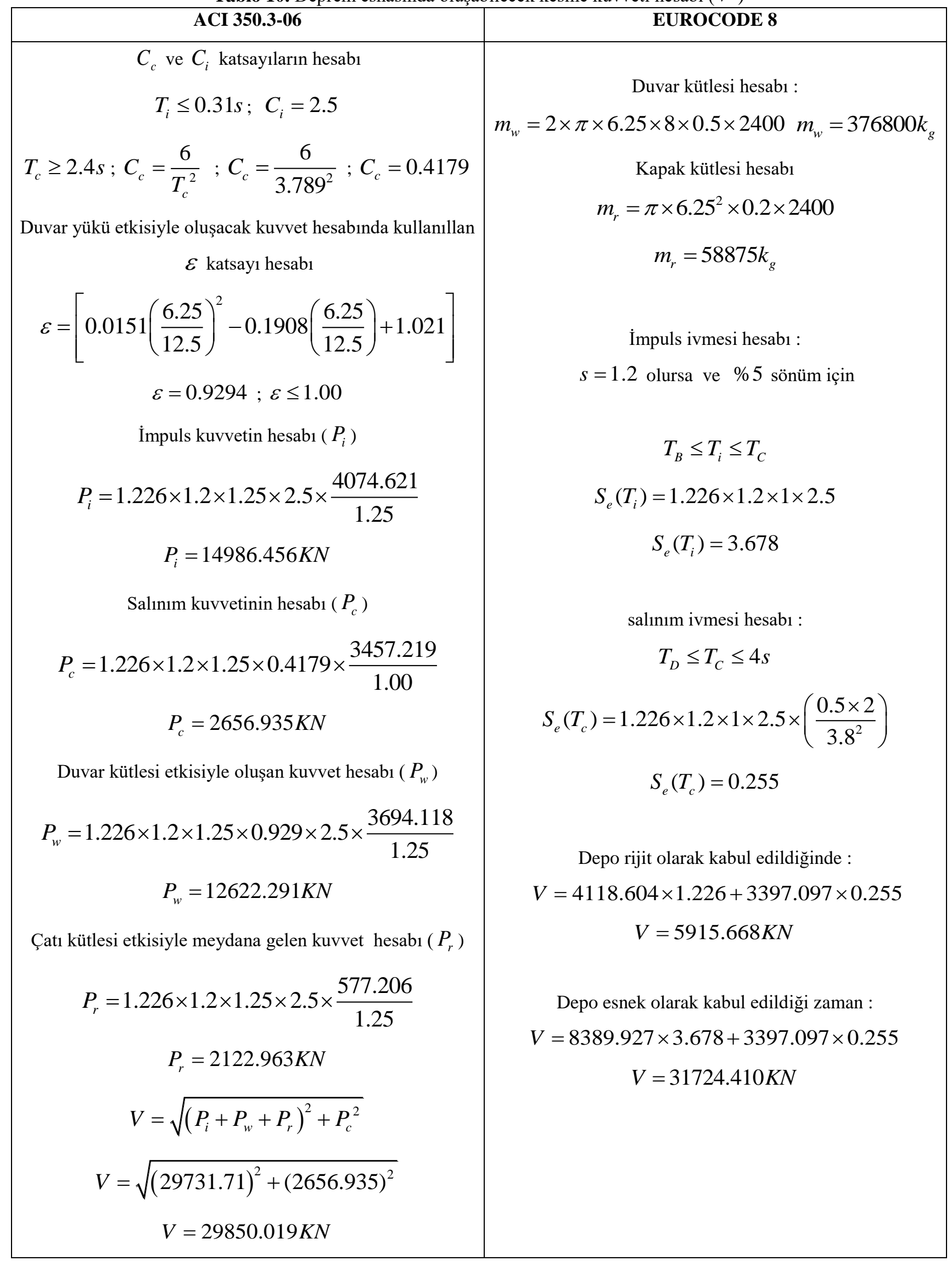


Tablo 11. Deprem esnasında oluşabilecek Eğilme momentin hesabı $\left(M_{b}\right)$

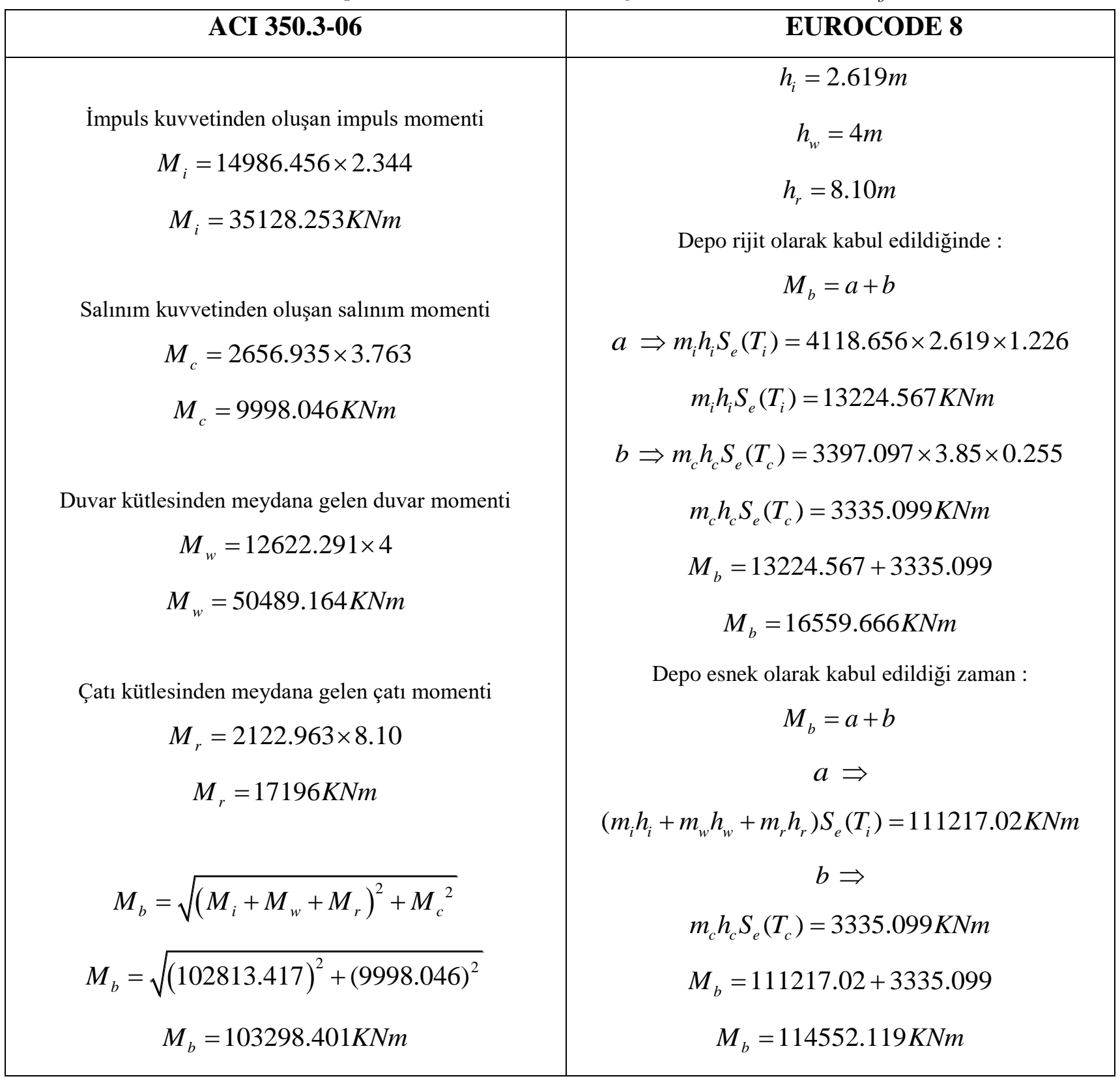

Tablo 12. Deprem esnasında oluşabilecek devirici momentin hesabı $\left(M_{o}\right)$

\begin{tabular}{|c|c|}
\hline ACI 350.3-06 & EUROCODE 8 \\
Devirici momenti hesabında kullanılan impuls \\
momenti & $c \Rightarrow$ \\
$M_{i}^{\prime}=14986.456 \times 4.981$ & $m_{i} h_{i}^{\prime} S_{e}\left(T_{i}\right)=4118.656 \times 4.506 \times 1.226$ \\
$M_{i}^{\prime}=74647.537 \mathrm{KNm}$ & $m_{i} h_{i}^{\prime} S_{e}\left(T_{i}\right)=22752.922 \mathrm{KNm}$ \\
& $d \Rightarrow$ \\
\hline
\end{tabular}




\begin{tabular}{|c|c|}
\hline $\begin{array}{c}\text { Devirici momenti hesabında kullanılan salınım } \\
\text { momenti }\end{array}$ & $m_{c} h_{c} S_{e}\left(T_{c}\right)=3397.097 \times 4.906 \times 0.255$ \\
$M_{c}^{\prime}=2656.935 \times 4.9$ & $m_{c} h_{c}^{\prime} S_{e}\left(T_{c}\right)=4249.870 \mathrm{KNm}$ \\
$M_{c}^{\prime}=13018.981 K N m$ & $M_{o}=22752.922+4249.870$ \\
& $M_{o}=27002.792 \mathrm{KNm}$ \\
$M_{o}=\sqrt{\left(M_{i}^{\prime}+M_{W}+M_{r}\right)^{2}+M_{c}^{\prime 2}}$ & Depo esnek olarak kabul edildiği zaman : \\
$M_{o}=\sqrt{(142332.701)^{2}+(13018.981)^{2}}$ & $M_{o}=c+d$ \\
$M_{o}=142926.875 K N m$ & $c \Rightarrow$ \\
& $\left(m_{i} h_{i}^{\prime}+m_{w} h_{w}+m_{r} h_{r}\right) S_{e}\left(T_{i}\right)=139802.58 K N m$ \\
$d \Rightarrow$ & $\Rightarrow$ \\
& $m_{c} h_{c}^{\prime} S_{e}\left(T_{c}\right)=4249.870 K N m$ \\
& $M_{o}=139802.58+4249.870$ \\
& $M_{o}=144052.45 \mathrm{KNm}$
\end{tabular}

Tablo 13. Maksimum oluşabilecek dalga yüksekliğin hesabı $\left(d_{\max }\right)$

\begin{tabular}{|c|c|}
\hline ACI 350.3-06 & EUROCODE 8 \\
\hline$d_{\text {max }}=\left(\frac{12.5}{2}\right) \times 1.25 \times 1 \times \frac{1.226 \times 0.255}{3.8^{2}}$ & $d_{\max }=6.25 \times \frac{0.255}{9.81}$ \\
$d_{\text {max }}=0.174 m$ & $d_{\max }=0.162 \mathrm{~m}$ \\
\hline
\end{tabular}

\section{SONUÇLARIN İRDELENMESI}

Gerçekleştirilen dinamik analizden ACI 350 ve EUROCODE 8 göre gerçekleştirilen çözümlemelerden hesaplanan kesme kuvvetinin karşılaştırılması Şekil 7 de, Eğilme momentinin karşılaş̧ırılması Şekil 8 de, devirici momentin karşılaştırılması Şekil 9 da, dalga yüksekliklerinin karşılaştırılması ise Şekil 10 da sunulmaktadır.

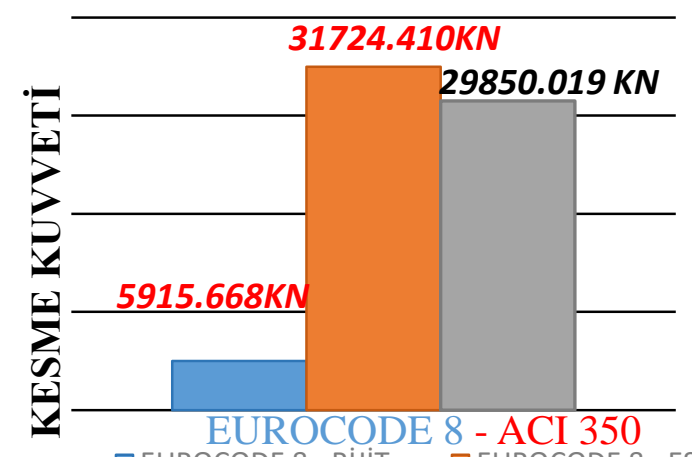

口EUROCODE 8 - RIJIT $\quad$ EUROCODE 8 - ESNEK

Şekil 7. Kesme kuvvetinin karşılaştırılması

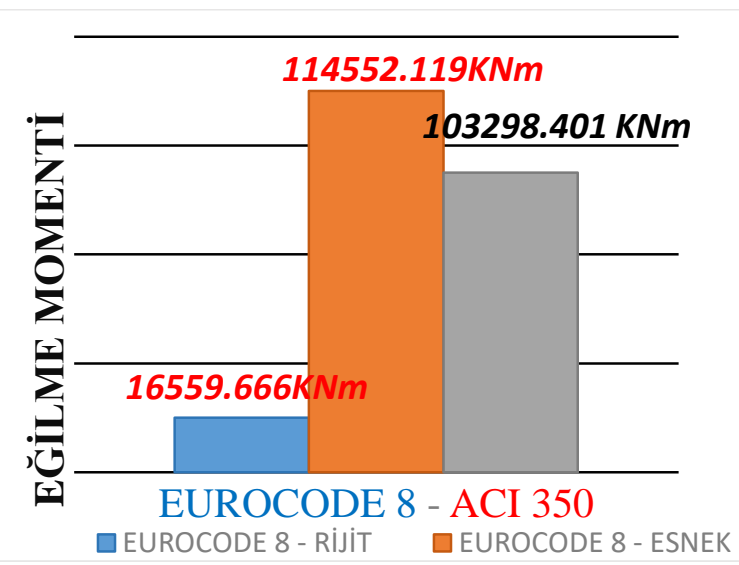

Şekil 8. Eğilme momentinin karşılaştırılması 


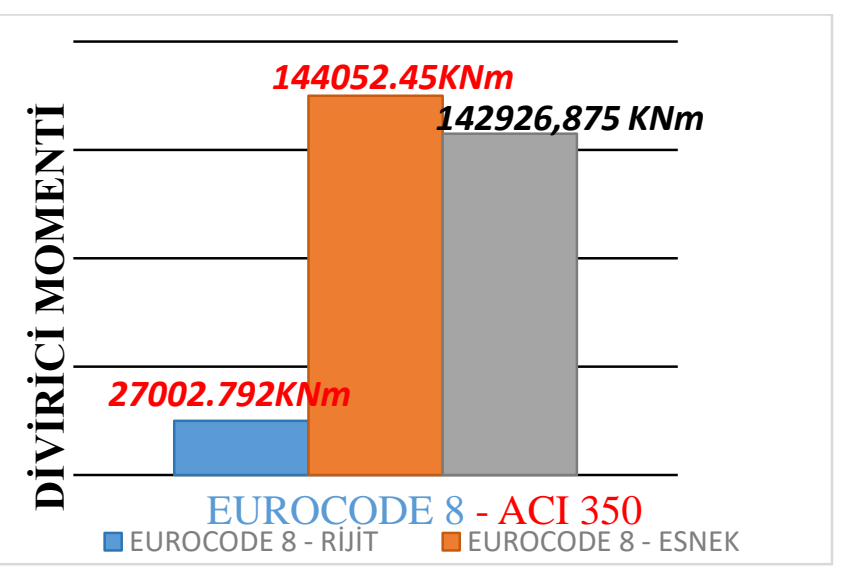

Şekil 9. Devirici momentin karşılaştırılması

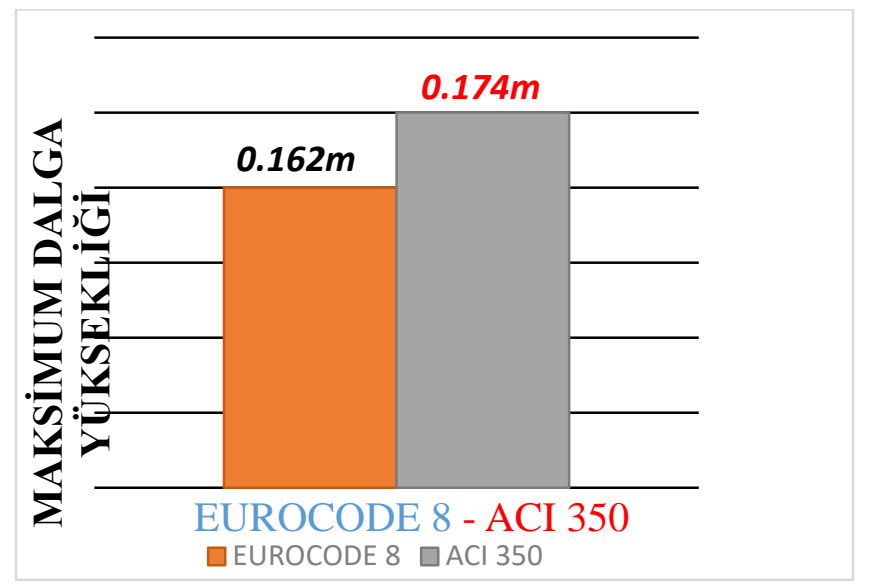

Şekil 10. Maksimum dalga yüksekliklerinin karşılaştırılması

\section{SONUÇ}

Çalışma kapsamında ACI 350 ve EUROCODE 8'de silindirik sıvı depoları için öngörülen bağıntılar karşılaştırılmalı olarak sunulmuştur. Örnek bir silindirik depo seçilerek belirtilen iki koda göre depreme göre hesabı yapılarak dinamik parametreleri belirlenmiştir. Buna göre;

Eurocode 8'e göre gerçekleştirilen rijit ve esnek çözümler arasında çok büyük farklar çıkmaktadır. Dolayısıyla depoyu rijit olarak Kabul ederek gerçekleştirilen çözümlemeler gerçekte elverişsiz durumda kalabilmektedir.

Elde edilen impuls ve salınım kütleleri ile bunların etkime yükseklikleri birbirine yakın olarak belirlenmiş aralarındaki maksimum fark $\% 4$ olmuştur.

Kesme kuvveti, eğilme momenti, sıvı deposunun devirici momenti ve deprem esnasında oluşacak maksimum dalga yüksekliğinin değerleri de birbirine yakın olarak belirlenmiştir. Maksimum fark \%6 olmuştur.

\section{KAYNAKÇA}

[1]. Eurocode 8, "Design of structures for earthquake resistance", Part 4: Silos, Tanks and Pipelines, Brussels, Belgium, no. prEN1988-4, pp. 44-81, 2006.

[2].Eurocode 8, "Design of structures for earthquake resistance", Part 1: General Rules, Seismic Actions and Rules fot Buildings, Ground Conditions and Seismic Action, Brussels, Belgium, no. EN1988-1, pp. 33-44, 2004.

[3].ACI 350.3-06, "American concrete institute committee ", Seismic design of liquid containing concrete structures and Commentary, America, 1-63, 2006.

[4].P.K. Malhotra, T. Wenk, and M. Wieland, "Simple procedure for seismic of liquid storage tanks", Structural engineering international, 10 (3), 197-201, (2010).

[5].M. Faridkhouri and W.J. Elias, "Determination of hydrodynamic forces in rectangular storage tanks unders seismic action ", International Journal of Scientific Research and Reviews, 4 (1), 50-67, (2015).

[6].V. Vukobratovic and D. Ladinovic, "A simplified seismic analysis of simplified seismic analysis of circular liquid storage tanks ", The $6^{\text {th }}$ psu-uns international conference on engineering and technology, Novi sad, Serbia, 2013.

[7].F. Majid, M. Lahlou, M. El ghorba, and A. Hachim, "Réservoirs de stockage : Méthodologie de calcul et analyse sécuritaire", $22^{\text {éme }}$ congres français de mécanique, Paris, France, 2015.

[8].K. Meskouris, B. Holtschoppen, C. Butenweg, and J. Rosin, "Seismic analysis of liquid storage tanks", $2^{\text {nd }}$ INQUA-IGCP-567 International Workshop on Active Tectonics, Earthquake Geology, Archaeology and Engineering, Corinth, Greece, 2011.

[9].A. Doğangün, "Betonarme sıvı depoları ve projelendirme ilkeleri", Yüksek Lisans Tezi, KTÜ Fen Bilimleri Enstitüsü, Trabzon, Türkiye, 1989.

[10]. A. Doğangün, "Dikdörtgen kesitli su depolarının sonlu elemanlar yöntemiyle depo-Sıvı zemin etkileşimini dikkate alarak Analitik yöntemlerle karşılaştırmalı deprem analizi ", Doktora Tezi, KTÜ Fen Bilimleri Enstitüsü, Trabzon, Türkiye, 1995.

[11]. R. Livaoğlu, "Ayaklı depoların sıvı-yapı-zemin etkileşimleri dikkate alınarak deprem davranışlarının incelenmesi ", Doktora Tezi, KTÜ Fen Bilimleri Enstitüsü, Trabzon, Türkiye, 2005.

[12]. Y. Yildız, "Betonarme siv1 depolarının farklı yönetmeliklere göre karşılaştırmalı deprem analizi ", Yüksek Lisans Tezi, KTÜ Fen Bilimleri Enstitüsü, İnşaat mühendisliği Anabilim Dalı, Trabzon, Türkiye, 2009.

[13]. I. T. Avval, "Dynanic response of concrete rectangular liquid tanks in three-dimensional space", Ph. D. thesis, Ryerson University, Toronto, Canada, 2012. 